\title{
Características de carcaça e componentes de peso vivo de bezerros recebendo diferentes dietas líquidas
}

\section{Carcass characteristics and live weight components of calves receiving different liquid diets}

\author{
Patrícia de Oliveira Lima ${ }^{\mathrm{I}^{*}}$ Magno José Duarte Cândido ${ }^{\mathrm{II}}$ \\ Antonia Lucivânia de Sousa Monte ${ }^{\mathrm{III}}$ Renata Nayhara de Lima ${ }^{\mathrm{I}}$ \\ Maria Vivianne Freitas Gomes de Miranda ${ }^{I}$ Rebeca Magda da Silva Aquino ${ }^{I I}$ \\ Rennan Herculano Rufino Moreira ${ }^{I I}$ Hélia Maria de Souza Leite ${ }^{I}$
}

RESUMO

Objetivou-se neste trabalho medir a influência de diferentes dietas líquidas sobre o rendimento de carcaça, cortes comerciais e componentes do peso vivo de bezerros mestiços, abatidos aos 60 dias de idade. Foram utilizados 24 bezerros mestiços, distribuídos em um delineamento inteiramente casualizado com quatro tratamentos e seis repetições: Leite integral (Controle); 50\% Leite integral $+50 \%$ de Soro de queijo in natura; $50 \%$ Leite integral $+50 \%$ de Soro de queijo adicionado de um ovo integral; e 50\% Leite integral $+50 \%$ de Soro de queijo adicionado de um ovo integral, adicionado de biotina. Foram avaliados o consumo, ganho de peso e todos os dados relacionados às características e rendimentos de carcaça, rendimentos dos cortes comerciais e componentes de peso vivo. Os dados foram submetidos à análise de variância e teste de comparação de médias Tukey $(P<0,05)$. Não foram observadas diferenças significativas entre os tratamentos testados sob as variáveis avaliadas. A substituição do leite integral por soro de queijo in natura pode ser adotada como forma de reduzir o custo de produção na fase de aleitamento, uma vez que tal substituição não interfere negativamente no desenvolvimento dos animais.

Palavras-chave: área de olho de lombo, biotina, ganho de peso, ovo integral, soro de queijo.

\section{ABSTRACT}

This research evaluated the influence of different liquid diets on carcass yield, and components of retail cuts weight of crossbred calves slaughtered at 60 days of age. It was used 24 crossbred calves distributed in a completely randomized design with four treatments and six replications: whole milk, 50\% whole milk $+50 \%$ of cheese whey, $50 \%$ whole milk $+50 \%$ cheese whey supplemented with a whole egg; whole milk and 50\% + 50\% of cheese whey supplemented with a egg added with biotin. It was evaluated intake, weight gain and slaughter weight. After slaughter at 60 days, it was collected all data related to carcass characteristics, commercial cut yields and components of body weight. The data were analyzed using ANOVA and Tukey mean comparison $(P<0.05)$. The substitution of milk by cheese whey in nature can be adopted as a way to reduce the cost of production in lactating since such a substitution will not adversely interfere with the development of animals.

Key words: biotin, cheese whey, rib eye area, weight gain, whole egg.

\section{INTRODUÇÃO}

Na maioria das propriedades leiteiras, os machos continuam sendo um problema para o produtor, por concorrer com a própria atividade, pois necessitam ingerir quantidades significativas de leite na fase inicial do seu desenvolvimento, tornandose fator limitante à criação, sendo aconselhável a substituição, o mais rápido possível, do leite por outros alimentos líquidos. Segundo FONTES et al. (2006), dentre os substitutos lácteos de origem animal, encontra-se o soro de queijo, cujas proteínas apresentam elevado valor biológico e sua utilização permite ainda a redução da liberação de resíduos poluentes no meio ambiente e o aumento na margem de lucratividade da indústria. De acordo com LIMA et al. (2011), a substituição total do leite por soro é impossível por não permitir a correta nutrição de

'Departamento de Ciências Animais, Universidade Federal Rural do Semiárido (UFERSA), 59625-900, Mossoró, RN, Brasil. E-mail: pattlima@bol.com.br. *Autor para correspondência.

"Departamento de Zootecnia, Universidade Federal do Ceará (UFC), Fortaleza, CE, Brasil.

IIIInstituto Federal do Ceará (IFCE), Limoeiro do Norte, CE, Brasil. 
bezerros em fase de amamentação. Uma alternativa complementar para as proteínas do soro pode ser a inclusão do ovo, por ser considerado um alimento completo, uma vez que, segundo LEHNINGER et al. (2000), contém todos os nutrientes necessários para produzir um novo organismo. Além disso, o ovo é utilizado comumente pelos produtores para formular dietas líquidas para alimentar crias que não tem o aleitamento natural. No entanto, a presença de fatores antinutricionais, como a avidina, a qual forma um complexo irreversível com a biotina (QUIGLEY, 2002), pode tornar necessária a suplementação dessa vitamina.

Além dos altos custos na fase de cria, a manutenção até a idade de abate acarreta na ocupação de espaço nos pastos, gerando concorrência com vacas em lactação. Portanto, uma alternativa é a criação desses bezerros para a produção de carne. Ressalta-se, porém, que são escassos os trabalhos que contemplem esse propósito, especialmente quando se trata de bovinos mestiços de origem leiteira, sobretudo quando abatidos superjovens. O rendimento de carcaça e dos cortes comerciais são medidas de interesse dos frigoríficos na avaliação do valor do produto adquirido e nos custos operacionais, visto que carcaças com pesos diferentes demandam a mesma mão-de-obra e tempo de processamento (COSTA et al., 2002). MELO et al. (2007) afirmam que o estudo do rendimento de cortes cárneos é importante, uma vez que complementa as informações técnicas das características de carcaça dos diferentes grupos genéticos de bovinos.

Objetivou-se neste trabalho medir a influência de diferentes dietas líquidas sobre o rendimento de carcaça, cortes comerciais e componentes do peso vivo de bezerros mestiços abatidos aos 60 dias de idade.

\section{MATERIAL E MÉTODOS}

Foram utilizados 24 bezerros mestiços (3/4 holandês e 1/4 zebu SPRD) que receberam diariamente quatro litros de leite integral, em dois fornecimentos, pela manhã e à tarde, após a fase de colostro (zero a três dias). Os animais do tratamento controle permaneceram nessa dieta até o abate, aos 60 dias de idade. Já os demais tratamentos receberam o leite integral até o $10^{\circ}$ dia e passaram por um período de adaptação de dez dias, que antecedeu o início dos respectivos tratamentos, quando foram distribuídos em um delineamento inteiramente casualizado com quatro tratamentos e seis repetições: $100 \%$ Leite integral (Controle); 50\% Leite integral $+50 \%$ de Soro de queijo in natura; $50 \%$ Leite integral $+50 \%$ de Soro de queijo in natura + um ovo integral in natura; e 50\% Leite integral $+50 \%$ de Soro de queijo in natura + um ovo integral in natura + biotina (5g). Em todos os tratamentos, os animais tiveram à sua disposição cochos contendo concentrado comercial para bezerro e feno de Tifton-85 (Cynodon sp.), além de água ad libitum, desde o nascimento até o desmame, que ocorreu aos 60 dias de idade.

O desempenho ponderal dos animais foi mensurado através de pesagens semanais até o desaleitamento, sempre pela manhã e antes do fornecimento da dieta líquida. Para determinação do consumo de matéria seca, foram feitas medições de consumo de feno e concentrado, diariamente, pelo método da oferta/sobra, com o ajuste da quantidade fornecida de forma a atingir um percentual de $10 \%$ de sobra em relação ao consumo efetuado no dia anterior. Diariamente, retiraram-se alíquotas das sobras para constituírem amostra composta semanal para posterior análise químico-bromatológica, cujos resultados foram: feno de Tifton-85 (94,18\% MS; 8,79\% MM; 7,83\% PB; 0,92\% EE; 83,47\% FDN e; $37,69 \%$ FDA) e para o concentrado (92,18\% MS; 10,40\% MM; 22,69\% PB; 4,42\% EE; 9,47\% FDN e; 4,60\% FDA).

Antes de serem abatidos, aos 60 dias de idade, os bezerros foram pesados e submetidos a um jejum de aproximadamente 16 horas, tendo acesso somente à água. $\mathrm{O}$ abate se deu em abatedouro privado, mantido sob fiscalização do Serviço de Inspeção Estadual (SIE) na cidade de Fortaleza-CE. Os bezerros foram esfolados e as patas foram retiradas, seguindo-se a evisceração, quando se recolheram todas as partes não-integrantes da carcaça para que se procedesse ao estudo biométrico. Foram observadas, de acordo com PEROBELLI (1993), as seguintes variáveis relativas às características quantitativas de carcaça: peso de carcaça quente - peso (kg) obtido logo após o abate; peso de carcaça fria - peso $(\mathrm{kg})$ obtido após 24h em câmara fria; peso de corpo vazio obtido diretamente pelo somatório dos pesos de patas, cabeça, pele, aparelho reprodutor, sangue, órgãos, vísceras vazias, gordura interna e carcaça; perda de peso por resfriamento - diferença $(\mathrm{kg})$ entre o peso de carcaça quente e o peso de carcaça fria; rendimento de carcaça quente - relação percentual entre o peso do corpo vazio e o peso de carcaça quente; rendimento de carcaça fria - relação percentual entre o peso do 
corpo vazio e o peso de carcaça fria; e quebra ao resfriamento - relação percentual entre o peso de carcaça quente e o peso de carcaça fria.

Na carcaça resfriada, foram realizados os cortes retirando-se o pescoço, as paletas, costelas e pernas (direitas e esquerdas), os filés, o lombo e o rabo. Em seguida, foi feita a desossa, resultando nos principais cortes comerciais, seguindo a metodologia sugerida por PARDI et al. (1993). Elas foram pesadas e registradas, assim como a gordura total e ossos resultantes dos cortes. Antes que houvesse a desossa do lombo, foi tomada a área da face do músculo Longissimus dorsi, resultante do corte transversal, feito entre a $12^{a}$ e $13^{\text {a }}$ costelas, através do desenho de seus contornos, em quatro repetições, obtidos com o auxílio de uma transparência. Dessas repetições, tomou-se a média da maior altura (A) e do maior comprimento (B) para cálculo da área de olho-delombo, segundo a fórmula: $\mathrm{AOL}=(\mathrm{A} / 2 \times \mathrm{B} / 2)$.

Os dados foram submetidos à análise de variância e teste de comparação de médias, utilizando o pacote estatístico SAS (SAS Institute Inc., North Carolina). Os efeitos dos diferentes tratamentos sobre cada variável foram comparados por meio do teste de Tukey, em nível de 5\% de probabilidade.

\section{RESULTADOS E DISCUSSÃO}

As dietas líquidas testadas não influenciaram no consumo de matéria seca (CMS) e no ganho médio diário (GMD) dos animais $(\mathrm{P}>0,05)$, conforme apresentado na tabela 1. Resultados semelhantes foram observados por VASCONCELOS et al. (2009), testando diferentes dietas líquidas na alimentação de bezerros. Já TEIXEIRA et al. (2007) não verificaram diferenças no ganho médio diário de bezerros recebendo sucedâneo a base de soro de queijo em substituição ao leite $\left(0,300 \mathrm{~kg} \mathrm{dia}^{-1}\right.$ e $0,250 \mathrm{~kg}$ $\mathrm{dia}^{-1}$, respectivamente). Ao avaliarem o desempenho de bezerros alimentados com sucedâneos a base de soro de leite, FONTES et al. (2006) observaram que a substituição em até $64,4 \%$ do leite por soro não afetou o consumo de alimentos e o ganho em peso dos animais. No entanto, a substituição do leite com porcentagens superiores a esse valor afetou negativamente, e as piores médias para as variáveis de desempenho foram observadas quando da substituição de $100 \%$ do leite integral. Esses mesmos autores constataram, com relação ao consumo de matéria seca, que, embora não significativo, pode-se observar uma correlação entre o consumo e o nível de substituição do leite integral por soro de queijo. Os dados apurados neste trabalho sugerem que o nível de substituição adotado não foi suficientemente elevado ao ponto de causar transtornos digestivos que refletissem sobre o consumo de matéria seca e ganho de peso.

As análises de variância não evidenciaram a existência de interferência das dietas líquidas para qualquer variável de característica de carcaça estudada, o que confirma os resultados encontrados para o CMS e GMD. As médias encontradas nos

Tabela 1 - Médias e coeficiente de variação (CV) das variáveis de desempenho, características de carcaça e rendimentos do abate de bezerros recebendo diferentes dietas líquidas.

\begin{tabular}{|c|c|c|c|c|c|}
\hline \multirow{2}{*}{ Variável } & \multirow[b]{2}{*}{ LI } & \multirow[b]{2}{*}{ LS } & \multirow[b]{2}{*}{ LSO } & \multirow[b]{2}{*}{ LSOB } & \multirow{2}{*}{$\mathrm{CV}$} \\
\hline & & & & & \\
\hline Consumo médio de matéria seca (g) & $406,7^{\mathrm{a}}$ & $520,8^{\mathrm{a}}$ & $429,3^{\mathrm{a}}$ & $446,5^{\mathrm{a}}$ & 33,3 \\
\hline Ganho médio diário (kg) & $0,39^{\mathrm{a}}$ & $0,41^{\mathrm{a}}$ & $0,34^{\mathrm{a}}$ & $0,32^{\mathrm{a}}$ & 23,7 \\
\hline Peso vivo pré-abate (kg) & $53,0^{\mathrm{a}}$ & $54,0^{\mathrm{a}}$ & $54,8^{\mathrm{a}}$ & $53,1^{\mathrm{a}}$ & 10,8 \\
\hline Peso vivo de abate (jejum) (kg) & $50,5^{\mathrm{a}}$ & $51,2^{a}$ & $52,6^{\mathrm{a}}$ & $50,6^{\mathrm{a}}$ & 11,1 \\
\hline Peso do corpo vazio (kg) & $44,6^{\mathrm{a}}$ & $45,1^{\mathrm{a}}$ & $46,4^{\mathrm{a}}$ & $41,7^{\mathrm{a}}$ & 15,3 \\
\hline Peso da carcaça quente (kg) & $26,9^{\mathrm{a}}$ & $26,3^{a}$ & $27,0^{\mathrm{a}}$ & $23,7^{\mathrm{a}}$ & 15,7 \\
\hline Peso da carcaça fria (kg) & $26,2^{\mathrm{a}}$ & $25,7^{\mathrm{a}}$ & $26,2^{\mathrm{a}}$ & $23,1^{\mathrm{a}}$ & 15,9 \\
\hline Perda por esfriamento (kg) & $0,72^{\mathrm{a}}$ & $0,52^{\mathrm{a}}$ & $0,77^{\mathrm{a}}$ & $0,70^{\mathrm{a}}$ & 29,6 \\
\hline Quebra por resfriamento (\%) & $2,67^{\mathrm{a}}$ & $2,49^{\mathrm{a}}$ & $2,58^{\mathrm{a}}$ & $2,46^{\mathrm{a}}$ & 26,6 \\
\hline Rendimento de carcaça quente (\%) & $53,5^{\mathrm{a}}$ & $51,3^{\mathrm{a}}$ & $50,5^{\mathrm{a}}$ & $49,2^{\mathrm{a}}$ & 5,1 \\
\hline Rendimento de carcaça fria (\%) & 52,0 & 50,2 & 49,0 & 47,8 & 5,0 \\
\hline Área de olho de lombo $\left(\mathrm{cm}^{2}\right)$ & $4,69^{\mathrm{a}}$ & $3,83^{\mathrm{a}}$ & $3,52^{\mathrm{a}}$ & $3,55^{\mathrm{a}}$ & 26,1 \\
\hline
\end{tabular}

a Valores com sobrescritos diferentes em uma mesma linha são estatisticamente diferentes, de acordo com o teste de Tukey (P<0,05); LI: Leite integral; LS: 50\% Leite integral + 50\% de Soro de queijo in natura; LSO: LS + ovo integral in natura; LSOB: LSO + biotina. 
diversos tratamentos, para peso vivo pré-abate sem jejum, peso vivo de abate com jejum e peso do corpo vazio apresentaram valores abaixo dos obtidos por outros autores. VASCONCELOS et al. (2009) e CARVALHO et al. (2003a) encontraram média de peso vivo ao abate de $52,21 \mathrm{~kg}$ e $53 \mathrm{~kg}$ para bezerros aos 50 dias de vida recebendo diferentes tipos de sucedâneos, respectivamente. Os menores pesos neste estudo podem dever-se em parte à utilização de animais mestiços, pois se sabe que os animais de raças taurinas puras apresentam notada diferença de precocidade em relação aos das raças zebuínas e seus mestiços, apresentando, portanto, crescimento e desenvolvimento mais acelerados, logo, maiores pesos ao abate.

Segundo VAZ et al. (2008), as características quantitativas das carcaças de bovinos são afetadas, principalmente, pelo peso de abate dos animais. Este representa o desenvolvimento corporal muscular, gorduras e esqueleto, que compõem a carcaça. Dessa forma, a similaridade verificada nos pesos e rendimentos de carcaça neste estudo é explicada pelo desenvolvimento semelhante dos animais, diferente dos resultados obtidos por CASTRO et al. (2004), que verificaram diferenças para pesos das carcaças, quente e fria, no estudo da interação entre dieta líquida e zeranol em animais abatidos aos 60 dias.

As perdas por resfriamento verificadas no presente estudo foram menores que as esperadas e do que aquelas apresentadas por outros trabalhos, inclusive quando os animais abatidos eram de maior idade ou maturidade, quando se esperam perdas menores em virtude de apresentarem uma camada de gordura de cobertura maior (FERNANDES et al., 2011; ALMEIDA et al., 2004). Menores médias para as perdas e quebras ao resfriamento são desejáveis sob o aspecto produtivo e qualitativo, pois indicam baixa perda de peso por gotejamento durante o processo de resfriamento das carcaças. Neste estudo, as menores perdas observadas, assim como o não escurecimento da carcaça, em decorrência do processo de resfriamento, podem ter ocorrido devido ao fato de haverem sido submetidas à câmara de refrigeração, envoltas em sacos plásticos. Logo, uma vez que a análise do incremento atribuído ao uso do saco plástico, no custo dos procedimentos de abate, se justifique, pode-se lançar mão dessa ferramenta como meio de reduzir as referidas perdas no rendimento das carcaças, sobretudo quando do abate de animais jovens destinados ao mercado de vitelos.
Quanto à área de olho de lombo não foram encontrados dados de outras pesquisas em virtude de não haverem trabalhos publicados sobre esse indicador, na idade em que os animais foram abatidos, não havendo, portanto, referências para a discussão. No entanto, a similaridade dos tratamentos testados com o tratamento controle implica desenvolvimento muscular semelhante entre os animais, uma vez que a área de olho de lombo é normalmente considerada um indicador de desenvolvimento muscular (RIBEIRO et al., 2001). Este, por sua vez, seria alterado ou por diferença no consumo de matéria seca, que não foi verificado, ou por diferenças de precocidade entre os animais estudados por diferenças entre raças, por exemplo. No entanto, nenhum dos casos se aplica a este trabalho.

As médias dos cortes comerciais apresentados na tabela 2 também não foram influenciadas pelas dietas propostas neste estudo $(\mathrm{P}>0,05)$. Tal semelhança pode ser atribuída ao grau de desenvolvimento semelhante dos animais nos diferentes tratamentos, já que não houve influência das dietas sobre o peso vivo. Deve-se salientar que a escassez de trabalhos relacionados ao rendimento de carcaças que contemplem os cortes comerciais e, sobretudo, os cortes nobres, dificultou a discussão, principalmente porque as poucas referências sobre esse ponto relatam o rendimento de animais abatidos em idades mais tardias, provavelmente, devido ao incipiente mercado para carne de vitelos no atual cenário nacional. Além disso, os principais cortes básicos das carcaças de bovinos praticados no mercado brasileiro são o dianteiro, com cinco costelas, a ponta de agulha e o traseiro especial (BONILHA et al., 2007), ficando os cortes nobres a critério da rede de distribuidores e sujeitos à regionalização, especialmente da nomenclatura utilizada. Como neste estudo optou-se pelo processamento da carcaça ainda no abatedouro, não foi possível medir os cortes comerciais básicos, resultando apenas nos cortes nobres.

O rendimento dos cortes por categorias também não foi influenciado pelas dietas testadas, no entanto pode-se observar que os cortes agrupados na categoria de carne de segunda apresentaram maiores valores, quando comparados aos de carnes de primeira. Entretanto, deve-se salientar que nessa categoria os cortes são desossados, enquanto que uma boa parcela dos de segunda são comercializados com seus respectivos ossos de sustentação. Além disso, quando comparados os mesmos cortes e seus respectivos 
Tabela 2 - Médias e coeficiente de variação (CV) dos cortes comerciais de bezerros recebendo diferentes dietas líquidas.

\begin{tabular}{|c|c|c|c|c|c|}
\hline \multirow{2}{*}{ Cortes comerciais (kg) } & \multicolumn{5}{|c|}{ 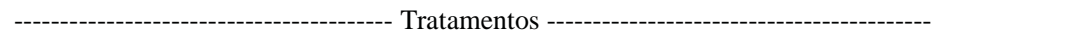 } \\
\hline & LI & LS & LSO & LSOB & \\
\hline Filé & $0,204^{\mathrm{a}}$ & $0,208^{\mathrm{a}}$ & $0,220^{\mathrm{a}}$ & $0,186^{\mathrm{a}}$ & 17,87 \\
\hline Alcatra + maminha & $0,453^{\mathrm{a}}$ & $0,480^{\mathrm{a}}$ & $0,464^{\mathrm{a}}$ & $0,431^{\mathrm{a}}$ & 17,09 \\
\hline Picanha & $0,151^{\mathrm{a}}$ & $0,152^{\mathrm{a}}$ & $0,150^{\mathrm{a}}$ & $0,145^{\mathrm{a}}$ & 12,35 \\
\hline Coxão mole & $0,952^{\mathrm{a}}$ & $0,913^{\mathrm{a}}$ & $0,938^{\mathrm{a}}$ & $0,804^{\mathrm{a}}$ & 15,19 \\
\hline Coxão duro & $0,878^{\mathrm{a}}$ & $0,848^{\mathrm{a}}$ & $1,111^{\mathrm{a}}$ & $0,746^{\mathrm{a}}$ & 31,41 \\
\hline Patinho & $0,564^{\mathrm{a}}$ & $0,589^{\mathrm{a}}$ & $0,591^{\mathrm{a}}$ & $0,519^{\mathrm{a}}$ & 14,12 \\
\hline Lombo & $0,896^{\mathrm{a}}$ & $0,906^{\mathrm{a}}$ & $0,986^{\mathrm{a}}$ & $0,831^{\mathrm{a}}$ & 18,22 \\
\hline Total de cortes de $1^{\mathrm{a}}(\mathrm{kg})$ & 4,098 & 4,096 & 4,460 & 3,662 & \\
\hline Total de cortes de $1^{\mathrm{a}}(\%)$ & 39,51 & 39,20 & 41,00 & 37,52 & \\
\hline Paleta & $1,382^{\mathrm{a}}$ & $1,483^{\mathrm{a}}$ & $1,478^{\mathrm{a}}$ & $1,390^{\mathrm{a}}$ & 13,89 \\
\hline Ossobuco dianteiro & $0,661^{\mathrm{a}}$ & $0,675^{\mathrm{a}}$ & $0,722^{\mathrm{a}}$ & $0,671^{\mathrm{a}}$ & 9,88 \\
\hline Ossobuco traseiro & $0,952^{\mathrm{a}}$ & $0,956^{\mathrm{a}}$ & $0,989^{\mathrm{a}}$ & $0,950^{\mathrm{a}}$ & 8,26 \\
\hline Pescoço & $1,159^{\mathrm{a}}$ & $1,105^{\mathrm{a}}$ & $1,122^{\mathrm{a}}$ & $1,119^{\mathrm{a}}$ & 12,19 \\
\hline Costela & $1,589^{\mathrm{a}}$ & $1,592^{\mathrm{a}}$ & $1,590^{\mathrm{a}}$ & $1,442^{\mathrm{a}}$ & 16,93 \\
\hline Rabo & $0,150^{\mathrm{a}}$ & $0,157^{\mathrm{a}}$ & $0,164^{\mathrm{a}}$ & $0,182^{\mathrm{a}}$ & 16,05 \\
\hline Vazio & $0,380^{\mathrm{a}}$ & $0,392^{\mathrm{a}}$ & $0,359^{\mathrm{a}}$ & $0,344^{\mathrm{a}}$ & 16,52 \\
\hline Total de cortes de $2^{\mathrm{a}}(\mathrm{kg})$ & 6,273 & 6,360 & 6,424 & 6,098 & \\
\hline Total de cortes de $2^{\mathrm{a}}(\%)$ & 60,49 & 60,80 & 59,00 & 62,48 & \\
\hline Total (kg) & 10,37 & 10,46 & 10,88 & 9,76 & \\
\hline
\end{tabular}

${ }^{a}$ Valores com sobrescritos diferentes em uma mesma linha são estatisticamente diferentes, de acordo com o teste de Tukey (P<0,05); LI: Leite integral; LS: 50\% Leite integral + 50\% de Soro de queijo in natura; LSO: LS + ovo integral in natura; LSOB: LSO + biotina.

pesos, sob o ponto de vista da remuneração paga pela carne, a amplitude anteriormente apresentada em termos percentuais da meia carcaça sofre uma redução bastante pronunciada, uma vez que, mesmo em menor proporção, os cortes de primeira têm preço diferenciado e privilegiado em relação aos de segunda. Isso faz com que, mesmo em menor quantidade, haja uma melhor remuneração.

Não foram encontradas diferenças significativas $(\mathrm{P}>0,05)$ nos pesos dos componentes do peso vivo dos bezerros abatidos aos 60 dias nos tratamentos propostos (Tabela 3). As médias verificadas neste estudo apresentam-se próximas às obtidas por CARVALHO et al. (2003b) para bezerros da raça Holandesa, abatidos aos 50 dias de idade. Isso demonstra que as dietas proporcionaram desenvolvimento fisiológico semelhante, pois não foram verificadas diferenças entre os pesos dos órgãos, principalmente aqueles de intensa atividade metabólica, como o fígado, que seriam afetados em caso de desbalanço nutricional. Neste estudo, a proposta de substituição de leite integral pelo soro de queijo implica menor aporte nutricional aos animais, especialmente do percentual de proteína e, em menor proporção, da energia, contidos no soro de queijo. Logo, esperava-se que as diferentes densidades nutricionais implicassem diferenças tanto no peso vivo quanto nos pesos de seus componentes. No entanto, isso não se verificou, indicando que, provavelmente, houve compensação no consumo de alimentos sólidos, que deve ter favorecido a manutenção do aporte de nutrientes, uma vez que nenhum dos componentes de peso vivo estudados apresentou diferença estatística entre os tratamentos.

\section{CONCLUSÃO}

A substituição do leite integral por soro de queijo in natura pode ser adotada como forma de reduzir o custo de produção na fase de aleitamento, uma vez que tal substituição não interfere negativamente no desenvolvimento dos animais. As inclusões do ovo, como fonte de proteína suplementar, e da biotina não melhoram o desempenho, sendo, portanto, dispensadas.

\section{COMITÊ DE ÉTICA E BIOSSEGURANÇA}

Protocolo nº 147/2009 - Comissão de Ética no Uso de Animais (CEUA)/ Universidade Estadual Paulista (UNESP). 
Tabela 3 - Médias e coeficiente de variação (CV) dos componentes do peso vivo de bezerros recebendo diferentes dietas líquidas.

\begin{tabular}{|c|c|c|c|c|c|c|}
\hline \multirow{2}{*}{\multicolumn{2}{|c|}{ Componentes do peso vivo (kg) }} & & & & & $C V$ \\
\hline & & LI & LS & LSO & LSOB & \\
\hline \multicolumn{2}{|l|}{ Fígado } & $0,940^{\mathrm{a}}$ & $0,891^{\mathrm{a}}$ & $0,933^{\mathrm{a}}$ & $0,893^{\mathrm{a}}$ & 21,9 \\
\hline \multicolumn{2}{|l|}{ Rins } & $0,248^{\mathrm{a}}$ & $0,221^{\mathrm{a}}$ & $0,230^{\mathrm{a}}$ & $0,227^{\mathrm{a}}$ & 20,5 \\
\hline \multicolumn{2}{|l|}{ Baço } & $0,173^{\mathrm{a}}$ & $0,153^{\mathrm{a}}$ & $0,168^{\mathrm{a}}$ & $0,167^{\mathrm{a}}$ & 46,6 \\
\hline \multicolumn{2}{|l|}{ Coração } & $0,394^{\mathrm{a}}$ & $0,393^{\mathrm{a}}$ & $0,431^{\mathrm{a}}$ & $0,405^{\mathrm{a}}$ & 17,9 \\
\hline \multicolumn{2}{|l|}{ Língua } & $0,249^{a}$ & $0,276^{\mathrm{a}}$ & $0,275^{\mathrm{a}}$ & $0,254^{\mathrm{a}}$ & 13,1 \\
\hline \multicolumn{2}{|l|}{ Sangue } & $1,757^{\mathrm{a}}$ & $2,338^{a}$ & $2,308^{a}$ & $2,163^{\mathrm{a}}$ & 22,1 \\
\hline \multicolumn{2}{|l|}{ Pele } & $4,481^{\mathrm{a}}$ & $4,805^{\mathrm{a}}$ & $4,883^{\mathrm{a}}$ & $4,607^{\mathrm{a}}$ & 17,9 \\
\hline \multicolumn{2}{|l|}{ Cabeça } & $2,407^{\mathrm{a}}$ & $2,475^{\mathrm{a}}$ & $2,608^{\mathrm{a}}$ & $2,413^{\mathrm{a}}$ & 17,1 \\
\hline \multicolumn{2}{|l|}{ Patas } & $1,940^{\mathrm{a}}$ & $2,108^{a}$ & $2,184^{\mathrm{a}}$ & $2,044^{\mathrm{a}}$ & 13,0 \\
\hline \multicolumn{2}{|l|}{ Pulmão } & $0,884^{\mathrm{a}}$ & $0,632^{\mathrm{a}}$ & $0,650^{\mathrm{a}}$ & $0,679^{\mathrm{a}}$ & 28,2 \\
\hline \multicolumn{2}{|l|}{ Traquéia } & $0,355^{\mathrm{a}}$ & $0,388^{\mathrm{a}}$ & $0,389^{\mathrm{a}}$ & $0,358^{\mathrm{a}}$ & 32,5 \\
\hline \multicolumn{2}{|l|}{ Esôfago } & $0,090^{\mathrm{a}}$ & $0,104^{\mathrm{a}}$ & $0,091^{\mathrm{a}}$ & $0,096^{\mathrm{a}}$ & 43,7 \\
\hline \multicolumn{2}{|l|}{ Pâncreas } & $0,275^{\mathrm{a}}$ & $0,169^{\mathrm{a}}$ & $0,159^{\mathrm{a}}$ & $0,139^{\mathrm{a}}$ & 34,0 \\
\hline \multicolumn{2}{|c|}{ Diafragma } & $0,254^{\mathrm{a}}$ & $0,256^{\mathrm{a}}$ & $0,270^{\mathrm{a}}$ & $0,229^{\mathrm{a}}$ & 22,6 \\
\hline \multicolumn{2}{|c|}{ Aparelho reprodutor } & $0,101^{\mathrm{a}}$ & $0,109^{\mathrm{a}}$ & $0,104^{\mathrm{a}}$ & $0,107^{\mathrm{a}}$ & 31,6 \\
\hline \multicolumn{2}{|c|}{ Rúmen-retículo } & $5,305^{a}$ & $5,084^{a}$ & $4,533^{a}$ & $5,958^{a}$ & 23,38 \\
\hline \multicolumn{2}{|l|}{ Omaso } & $0,332^{a}$ & $0,422^{\mathrm{a}}$ & $0,507^{\mathrm{a}}$ & $0,400^{a}$ & 34,17 \\
\hline \multicolumn{2}{|c|}{ Abomaso } & $1,026^{a}$ & $1,197^{\mathrm{a}}$ & $1,258^{a}$ & $0,967^{\mathrm{a}}$ & 32,19 \\
\hline \multicolumn{2}{|l|}{ Bexiga } & $0,122^{a}$ & $0,134^{\mathrm{a}}$ & $0,107^{\mathrm{a}}$ & $0,094^{\mathrm{a}}$ & 20,2 \\
\hline \multicolumn{2}{|c|}{ Vesícula biliar } & $0,028^{a}$ & $0,035^{\mathrm{a}}$ & $0,039^{\mathrm{a}}$ & $0,039^{a}$ & 28,9 \\
\hline \multirow{2}{*}{ Gordura } & Omento & $0,068^{\mathrm{a}}$ & $0,090^{\mathrm{a}}$ & $0,071^{\mathrm{a}}$ & $0,069^{\mathrm{a}}$ & 62,4 \\
\hline & Perirenal & $0,095^{\mathrm{a}}$ & $0,097^{\mathrm{a}}$ & $0,072^{\mathrm{a}}$ & $0,101^{\mathrm{a}}$ & 44,6 \\
\hline
\end{tabular}

${ }^{\text {a }}$ Valores com sobrescritos diferentes em uma mesma linha são estatisticamente diferentes, de acordo com o teste de Tukey (P<0,05); LI: Leite integral; LS: 50\% Leite integral + 50\% de Soro de queijo in natura; LSO: LS + ovo integral in natura; LSOB: LSO + biotina.

\section{REFERÊNCIAS}

ALMEIDA, J.G.A. et al. Desempenho, características de carcaça e resultado econômico de cordeiros criados em creep feeding com silagem de grãos úmidos de milho. Revista Brasileira de Zootecnia, v.33, n.4, p.1048-1059, 2004. Disponível em: $<$ http://www.scielo.br/scielo.php?script=sci_arttext\&pid =S1516-35982004000400025 $>$. Acesso em: 23 dez. 2012. doi: 10.1590/S1516-35982004000400025.

BONILHA, S.F.M. et al. Efeitos da seleção para peso pósdesmama sobre características de carcaça e rendimento de cortes cárneos comerciais de bovinos. Revista Brasileira de Zootecnia, v.36, n.5, p.1275-1281, 2007. Disponível em: <http://www.scielo. br/scielo.php?pid=S1516-35982007000600008\&script=sci_ arttext>. Acesso em: 14 dez. 2012. doi: 10.1590/S151635982007000600008 .

CARVALHO, P.A. et al. Características quantitativas, composição física tecidual e regional da carcaça de bezerros machos de origem leiteira ao nascimento, 50 e 110 dias de idade. Revista Brasileira de Zootecnia, v.32, n.6, p.1476-1483, 2003a. Disponível em: <http://www.scielo.br/scielo.php?pid=s151635982003000600023\&script=sci_arttext $>$. Acesso em: 18 jan. 2013 . doi: 10.1590/S1516-35982003000600023.
CARVALHO, P.A. et al. Componentes do peso vivo e órgãos viscerais de bezerros machos de origem leiteira ao nascimento, 50 e 110 dias de vida. Revista Brasileira de Zootecnia, v.32, n.6, p.1469-1475, 2003b. Disponível em: <http://www.scielo.br/scielo. php?pid=s1516-35982003000600022\&script=sci_arttext $>$. Acesso em: 13 dez. 2012. doi: 10.1590/S1516-35982003000600022.

CASTRO, A.L.M. et al. Desempenho e rendimento de carcaças de bezerros alimentados com colostro fermentado, associado ao óleo de zeranol. Arquivo Brasileiro de Medicina Veterinária e Zootecnia, v.56, n.2, p. 193-202, 2004. Disponível em: <http://www.scielo.br/scielo.php?script=sci_arttext\&pid =S0102-09352004000200009>. Acesso em: 02 jan. 2013. doi: 10.1590/S0102-09352004000200009.

COSTA, E.C. et al. Desempenho de novilhos Red Angus superprecoces confinados e abatidos com diferentes pesos. Revista Brasileira de Zootecnia, v.31, n.1, p.129-138, 2002. Disponível em: <http://www.scielo.br/scielo.php?script=sci_artte xt\&pid=S1516-35982002000100015>. Acesso em: 22 dez. 2012. doi: 10.1590/S1516-35982002000100015.

FERNANDES, S.R. et al. Desmame precoce e a suplementação concentrada no peso ao abate e nas características de carcaça de 
cordeiros terminados em pastagem. Revista Brasileira de Saúde e Produção Animal, v.12, n.2, p.527-537, 2011.

FONTES, F.A.P.V. et al. Desempenho de bezerros alimentados com dietas líquidas à base de leite integral ou soro de leite. Arquivo Brasileiro de Medicina Veterinária e Zootecnia, v.58, n.2, p.212-219, 2006. Disponível em: <http://www.scielo. br/scielo.php?pid=S0102-09352006000200010\&script=sci arttext>. Acesso em: 22 dez. 2013. doi: 10.1590/S010209352006000200010 .

LEHNINGER, A.L. et al. Principles of biochemistry. 3.ed. New York: Worth Publishers, 2000. p.1255.

LIMA, R.N. et al. Avaliação econômica de dietas liquidas a base de soro de queijo in natura para bezerros. Revista Brasileira de Saúde e Produção Animal, v.12, n.1, p.14-21, 2011.

MELO, W.S. et al. Cortes nobres, componentes do peso vivo e órgãos viscerais de bovinos mestiços de origem leiteira em condições de pastejo, restrito ou “ad libitum”. Revista Brasileira de Ciências Agrárias, v.2, n.1, p.90-97, 2007.

PARDI, M. C. et al. Ciência, higiene e tecnologia da carne: tecnologia da sua obtenção e transformação. Goiânia: Universidade de Goiás, 1993. V.1, 586p.

PEROBELLI, Z.V. Estudo das carcaças e da carne de vacas de descarte das raças Charolês e Nelore. 1993. 70f. Dissertação
(Mestrado em Zootecnia) - Universidade Federal de Santa Maria, RS.

QUIGLEY, J.D. Effects of spray-dried whole egg and biotin in calf milk replacer. Journal of Dairy Science, v.85, n. 1, p.198203, 2002. Disponível em: <http://www.calfnotes.com/pdffiles/ CNman45.pdf $>$. Acesso em: 15 jan. 2013. doi: 10.3168/jds.S00220302(02)74068-X.

RIBEIRO, T.R. et al. Características da carcaça de bezerros holandeses para produção de vitelos recebendo dietas com diferentes níveis de concentrado. Revista Brasileira de Zootecnia, v.30, n.6, p.2154-2162, 2001. Disponível em: <http://www.scielo. br/scielo.php?pid=S1516-35982001000800026\&script=sci_ arttext>. Acesso em: 12 jan. 2012. doi: 10.1590/S151635982001000800026 .

TEIXEIRA P.A. et al. Avaliação de diferentes dietas sobre o desempenho de bezerros da raça Holandesa durante o período de aleitamento. Ciência e Agrotecnologia, v.31, p.1831-1837, 2007.

VASCONCELOS A.M. et al. Desempenho de bezerros leiteiros submetidos a diferentes dietas líquidas e instalações durante o período hibernal. Acta Veterinaria Brasilica, v.3, n.4, p.163-171, 2009.

VAZ, F.N. et al. Características de carcaça de novilhos Aberdeen Angus terminados em pastagem cultivada ou confinamento. Ciência Animal Brasileira, v.9, n.3, p.590-597, 2008. 